\title{
A BRAIDED SIMPLICIAL GROUP
}

\author{
JIE WU
}

\begin{abstract}
By studying braid group actions on Milnor's construction of the 1sphere, we show that the general higher homotopy group of the 3 -sphere is the fixed set of the pure braid group action on certain combinatorially described group. We also give certain representation of higher differentials in the Adams spectral sequence for $\pi_{*}\left(S^{2}\right)$.
\end{abstract}

\section{INTRODUCTION}

In this article, we study the homotopy groups by considering the braid group actions on simplicial groups. The point of view here is to establish a relation between the fixed set of braid group actions and the homotopy groups of the 3-sphere. We first recall a combinatorial description of the homotopy groups of the 3 -sphere in [11].

Let $F\left(x_{1}, \cdots, x_{n}\right)$ be the free group generated by the letters $x_{1}, \cdots, x_{n}$. Let $w\left(x_{1}, \cdots, x_{n}\right)=x_{i_{1}}^{\epsilon_{1}} \cdots x_{i_{t}}^{\epsilon_{t}}$ be a word. Given $a_{1}, \cdots, a_{n} \in F\left(x_{1}, \cdots, x_{n}\right)$, we write $w\left(a_{1}, \cdots, a_{n}\right) \in F\left(x_{1}, \cdots, x_{n}\right)$ for $a_{i_{1}}^{\epsilon_{1}} \cdots a_{i_{t}}^{\epsilon_{t}}$. The $n$-th $W$-group $G(n)$ is the quotient group of $F\left(x_{1}, \cdots, x_{n}\right)$ modulo the following relations:

$\left(\mathcal{R}_{1}\right)$ the product $x_{1} \cdots x_{n}$;

$\left(\mathcal{R}_{2}\right)$ the words $w\left(x_{1}, \cdots, x_{n}\right)$ that satisfy: $w\left(x_{1}, \cdots, x_{i-1}, 1, x_{i+1}, \cdots, x_{n}\right)=1$ for $1 \leq i \leq n$.

Relations $\mathcal{R}_{2}$ consist of all of words that will be trivial if one of the generators is replaced by the identity element 1 . The smallest normal subgroup of $F\left(x_{1}, \cdots, x_{n}\right)$ which contains relations $\mathcal{R}_{1}$ and $\mathcal{R}_{2}$ was determined as a subgroup of $F\left(x_{1}, \cdots, x_{n}\right)$ generated by certain systematic and uniform iterated commutators [11.

Theorem 1.1. [11] For $n \geq 3$, the homotopy group $\pi_{n}\left(S^{3}\right)$ is isomorphic to the center of $G(n)$.

A natural question arisen from Theorem 1.1 is how to give a group theoretical approach to the homotopy groups, that is how to understand the center of the group $G(n)$. There is a canonical braid group action on $G(n)$ which is induced by the

Research is supported in part by the Academic Research Fund of the National University of Singapore RP3992646. 
canonical braid group action on the free group $F\left(x_{1}, \cdots, x_{n}\right)$, namely

$$
\sigma_{i}\left(x_{j}\right)=\left\{\begin{array}{ccc}
x_{i+1} \quad \text { if } & j=i \\
x_{i+1}^{-1} x_{i} x_{i+1} & \text { if } & j=i+1 \\
x_{j} & & \text { otherwise }
\end{array}\right.
$$

for $1 \leq i \leq n-1$. These actions gives a canonical homomorphism from the braid group $B_{n}$ into the automorphism group of $G(n)$. Since Quillen's plus construction of the classifying space for the stable braid group is (up to homotopy type) the double loop space of the 3-sphere [2], these braid group actions do not seem occasional event. Fred Cohen therefore conjectured that the center of $G(n)$ is the fixed set of the braid group action on $G(n)$. We answer Cohen's question as follows. Let $K_{n}$ be the pure braid group, that is, $K_{n}$ is the normal divisor of $B_{n}$ generated by $\sigma_{1}^{2}$ (See [6]). Equivalently $K_{n}$ is the kernel of the canonical homomorphism from $B_{n}$ to the symmetric group $\Sigma_{n}$. In geometry, the group $K_{n}$ is the fundamental group of the configuration space $F\left(\mathbb{R}^{2}, n\right)$, where

$$
F(M, n)=\left\{\left(x_{1}, \cdots, x_{n}\right) \in M^{n} \mid x_{i} \neq x_{j} \text { for } i \neq j\right\}
$$

for any manifold $M$ (See [2]). Let $Z(G(n))$ be the center of $G(n)$.

Theorem 1.2. For $n \geq 4$, then

1) the center of $G(n)$ is the fixed set of the pure braid group action on $G(n)$ and so is $\pi_{n}\left(S^{3}\right)$;

2) the fixed set of the braid group action on $G(n)$ is the subgroup

$$
\{x \in Z(G(n)) \mid 2 x=0\} .
$$

We should point out that the determination of the fixed set of $K_{n}$-action on $G(n)$ by (combinatorial) group theoretic means seems beyond the reach of current techniques. On the other hand, braid group actions have largely studied in several areas such as group theory and low dimensional topology. Various problems arising from physics are related to braid group actions as well. Theorem 1.2 suggests that the homotopy groups play certain role for braid group actions. In the range in which $\pi_{*}\left(S^{3}\right)$ is known $(* \leq 55$, see [5, 9]), by homotopy theoretic means, we gain insight into these difficult group theoretic questions.

The article is organized as follows. In section 2, we study the braid group action on the Milnor's construction on the simplicial 1-sphere. A relation between the simplicial structure and the braid group action is given in Proposition 2.1. This relation is based on the direct calculation. Roughly the braid group action interchanges the 
faces together with conjugates. It is possible to have a more general theory to study group actions on simplicial groups, particularly simplicial group models for iterated loop spaces. But we only intend to investigate the most important example $F\left(S^{1}\right)$ in this article. After establishing the systematic relations between the braid group actions and the simplicial structure, braided simplicial groups are introduced in this section. Then we show that loop simplicial group and the Moore-Postnikov system of a braided simplicial group are braided. Theorem 2.9 and Proposition 2.10 give a relation between the fixed set of the braid group action and the homotopy groups for a general braided simplicial group. Theorem 1.2 follows from Lemma 2.8 and Theorem 2.13. In section 3, we study a braided representation of the Milnor's construction of the simplicial 1-sphere into a simplicial algebra. Theorem 3.8 gives certain representation of higher differentials in the Adams spectral sequence for $\pi_{*}\left(S^{2}\right)$.

The author would like to thanks Professors Fred Cohen and Jon Berrick for their helpful suggestions and kind encouragements.

\section{Braid Group Actions on $F\left(S^{1}\right)$}

2.1. Braided simplicial groups. Let $F\left(S^{1}\right)$ be Milnor's construction of the simplicial 1-sphere $S^{1}$. Then $F\left(S^{1}\right)_{n+1}=F\left(y_{0}, \cdots, y_{n}\right)$ is a free group generated by letters $y_{0}, \cdots, y_{n}$ with the following simplicial structure

$$
d_{j} y_{k}=\left\{\begin{array}{ccc}
y_{k-1} & \text { for } & j \leq k \\
1 & \text { for } & j=k+1 \\
y_{k} & \text { for } & j>k+1
\end{array}\right.
$$

and

$$
s_{j} y_{k}=\left\{\begin{array}{ccc}
y_{k+1} & \text { for } & j \leq k \\
y_{k} y_{k+1} & \text { for } & j=k+1 \\
y_{k} & \text { for } & j>k+1
\end{array}\right.
$$

for $0 \leq j \leq n+1$, where $y_{-1}=\left(y_{0} \cdots y_{n-1}\right)^{-1}$ in $F\left(S^{1}\right)_{n}$ (See [11, Lemma 4.1]). Let the braid group $B_{n+1}$ act on $F\left(S^{1}\right)_{n+1}=F\left(y_{0}, \cdots, y_{n}\right)$ in the usual way, that is,

$$
\sigma_{k}\left(y_{j}\right)=\left\{\begin{array}{ccc}
y_{k+1} & \text { if } & j=k \\
y_{k+1}^{-1} y_{k} y_{k+1} & \text { if } & j=k+1 \\
y_{j} & & \text { otherwise }
\end{array}\right.
$$

for $0 \leq k \leq n-1$. Let $\sigma_{-1}$ be an automorphism of $F\left(y_{0}, \cdots, y_{n}\right)$ defined by

$$
\sigma_{-1}\left(y_{0}\right)=y_{0}^{-1} y_{-1} y_{0}=y_{0}^{-1} y_{n}^{-1} \cdots y_{1}^{-1} \quad \sigma\left(y_{j}\right)=y_{j} \quad \text { for } \quad j>0 .
$$

The subgroup of the automorphism group of $F\left(y_{0}, \cdots, y_{n}\right)$ generated by $\sigma_{j}$ for $-1 \leq$ $j \leq n-1$ is the braid group $B_{n+2}$. By direct calculation, we have 
Proposition 2.1. The following identities hold for the braid groups action on $F\left(S^{1}\right)$ :

$$
d_{j} \sigma_{k}=\left\{\begin{array}{cc}
\sigma_{k-1} d_{j} & j \leq k \\
d_{k+2} & j=k+1 \\
d_{k+1} & j=k+2 \\
\sigma_{k} d_{j} & j>k+2 \\
\sigma_{k+1} s_{j} & j \leq k \\
\sigma_{k+1} \circ \sigma_{k} \circ s_{k+2} & j=k+1 \\
\sigma_{k} \circ \sigma_{k+1} \circ s_{k+1} & j=k+2
\end{array}\right.
$$

By this proposition, we give the following definition.

Definition 2.2. A simplicial group $G$ is called braided if there is a braid group $B_{n+1^{-}}$ action on $G_{n}$ for each $n$ such that the identities 11 and 2 are satisfied, where $B_{n+1}$ is considered as the braid group generated by $\sigma_{-1}, \sigma_{0}, \cdots, \sigma_{n-2}$.

Let $G$ be a simplicial group and let $N G$ be the Moore chain complex of $G$, that is,

$$
N G_{n}=\left\{x \in G_{n} \mid d_{j} x=1 \quad \text { for } \quad j>0\right\}
$$

. Let $\mathcal{Z}(G)$ and $\mathcal{B}(G)$ be the sets of cycles and boundaries of $G$, respectively, that is,

$$
\begin{gathered}
\mathcal{Z}_{n}(G)=\left\{x \in G_{n} \mid d_{j} x=1 \text { for all } j\right\}, \\
\mathcal{B}_{n}(G)=\left\{d_{0} x \mid x \in N_{n+1}(G)\right\} .
\end{gathered}
$$

By Moore's classical theorem [7], $\pi_{n}(G)=\mathcal{Z}_{n}(G) / \mathcal{B}_{n}(G)$. Let $G$ be a braided simplicial group. A subgroup $H$ of $G$ is called a braided subgroup if $\sigma(H) \subseteq H$ for any $\sigma \in B_{n+1}$, that is, $H$ is invariant under $B_{n+1}$-action.

Proposition 2.3. Let $G$ be a braided simplicial group. Then the subgroups $\mathcal{Z}(G)_{n}$ and $\mathcal{B}(G)_{n}$ of $G_{n}$ are braided. 
Proof. By Proposition 2.1, $\mathcal{Z}(G)_{n}$ is a braided subgroup. Now let $x=d_{0} y \in \mathcal{B}(G)_{n}$, where $y \in N G_{n+1}$. By Proposition 2.1, we have

$$
\sigma_{k} x=\sigma_{k} d_{0} y=d_{0} \sigma_{k+1} y
$$

for each $k \geq-1$ and

$$
d_{j} \sigma_{k+1} y=1
$$

for each $j>0$ and $k \geq-1$. Thus $\sigma_{k} x \in \mathcal{B}(G)_{n}$ for each $k \geq-1$ and so $\mathcal{B}(G)_{n}$ is a braided subgroup, which is the assertion.

Note: $N_{n}(G)$ is invariant under the subgroup of $B_{n+1}$ generated by $\sigma_{k}$ with $k \geq 0$. But it is not invariant under $\sigma_{-1}$.

Since there is a relation

$$
d_{0} \sigma_{-1}=d_{1}, \quad d_{1} \sigma_{-1}=d_{0}, \quad \text { and } \quad d_{j} \sigma_{-1}=\sigma_{-1} d_{j} \quad \text { for } \quad j>1,
$$

we have

Proposition 2.4. Let $G$ be a braided simplicial group. Then

$$
\mathcal{Z}(G)_{n}=N G_{n} \cap \sigma_{-1}\left(N G_{n}\right) .
$$

for each $n$.

Now we study the braided group actions on the Postnikov systems of a braided simplicial group $G$. Let $I=\left(i_{1}, \cdots, i_{k}\right)$ be a sequence of non-negative integers and let $d_{I}$ denote the composite of face homomorphisms

$$
d_{I}=d_{i_{1}} \cdots d_{i_{k}} .
$$

Given a simplicial group $G$, the simplicial sub groups $R_{n} G$ and $\bar{R}_{n}(G)$ are defined as follows:

$$
\begin{gathered}
R_{n} G_{q}=\left\{x \in G_{q} \mid d_{I}(x)=1 \quad \text { forany } \quad I=\left(i_{1}, \cdots, i_{q-n}\right)\right\}, \\
\bar{R}_{n} G_{q}=\left\{x \in G_{q} \mid d_{I}(x) \in \mathcal{B}(G)_{n} \text { forany } I=\left(i_{1}, \cdots, i_{q-n}\right)\right\} .
\end{gathered}
$$

Let $P_{n} G=G / R_{n} G$ and $\bar{P}_{n} G=G / \bar{R}_{n} G$. Then $\left\{P_{n} G\right\}$ is the Postnikov system of $G$ (See [4, 7]). The quotient homomorphism $P_{n} G \rightarrow \bar{P}_{n} G$ is a homotopy equivalence (See [1]). The tower

$$
\cdots \rightarrow P_{n} G \rightarrow \bar{P}_{n} G \rightarrow P_{n-1} G \rightarrow \cdots
$$

is called a modified Postnikov system of $G$. One of the important properties of the modified Postnikov system is that the short exact sequence of simplicial groups

$$
K\left(\pi_{n}(G), n\right) \rightarrow \bar{P}_{n} G \rightarrow P_{n-1} G
$$

is a central extension [11, Theorem 2.12].

By Propositions 2.1 and 2.3, we have 
Theorem 2.5. Let $G$ be a braided simplicial group. Then, for each $n$, the simplicial quotient groups $P_{n} G$ and $\bar{P}_{n} G$ are braided. Thus the modified Postnikov system of $G$ are braided. In particular, there is a braided central extension

$$
K\left(\pi_{n}(G), n\right) \rightarrow \bar{P}_{n}(G) \rightarrow P_{n-1}(G) .
$$

Let $G$ be a braided simplicial group. Then $R_{0} G$ is a braided simplicial subgroup of $G$ by the theorem above. Recall that the loop simplicial group $\Omega G$ of $G$ is defined by $\Omega G_{n}=\operatorname{Ker}\left(d_{0}\right) \cap R_{0} G_{n+1}$ with $d_{j}(\Omega G)=d_{j+1}(G)$ and $s_{j}(\Omega G)=s_{j+1}(G)$ (See 四). By Proposition 2.1, $\operatorname{Ker}\left(d_{0}\right) \cap R_{0} G_{n+1}$ is invariant under the action of $\sigma_{j}$ for $0 \leq j \leq n-1$. Let $B_{n+1}^{\prime}$ be the subgroup of $B_{n+2}$ generated by $\sigma_{0}, \cdots, \sigma_{n-1}$. Then $B_{n+1}^{\prime} \cong B_{n+1}$ under the canonical isomorphism which sends $\sigma_{j}$ to $\sigma_{j-1}$ for $0 \leq j \leq n-1$. Thus we obtained the following theorem.

Theorem 2.6. Let $G$ be a braided simplicial group. Then the loop simplicial group $\Omega G$ is braided. Thus any iterated loop simplicial groups of $G$ are braided.

Corollary 2.7. The loops and the modified Postnikov system of $F\left(S^{1}\right)$ are braided.

2.2. Fixed Sets of Braided Actions. Let $G$ be a simplicial group and let $x, y \in G_{n}$ be two elements. We call that $x$ is homotopic to $y$, which is denoted by $x \simeq y$, if $x y^{-1} \in \mathcal{B}(G)_{n}$.

Lemma 2.8. Let $G$ be a braided simplicial group and let $x \in \mathcal{Z}(G)_{n}$ be a cycle with $n \geq 1$. Then

$$
\sigma_{k}(x) \simeq x^{-1}
$$

for each $k \geq-1$.

Proof. For each $k \geq-1$, consider the element $\sigma_{k+1} s_{k+1} x$. By the identities 1 and 2, we have

$$
d_{j} \sigma_{k+1} s_{k+1} x=\left\{\begin{array}{cccc}
\sigma_{k} d_{j} s_{k+1} x=1 & \text { for } & j<k+1 \\
\sigma_{k} x & \text { for } & j=k+1 \\
d_{k+3} s_{k+1} x=1 & \text { for } & j=k+2 \\
d_{k+2} s_{k+1} x=x & \text { for } & j=k+3 \\
\sigma_{k+1} d_{j} s_{k+1} x=1 & \text { for } & j>k+3 .
\end{array}\right.
$$

Thus $\sigma_{k} x \simeq x^{-1}$, which is the assertion. 
Let $G$ be a braided simplicial group and let $S$ be a subset of $G_{n}$. Define

$$
B_{n+1}(S)=\left\{\sigma \in B_{n+1} \mid \sigma x \simeq x \text { for all } x \in S\right\} .
$$

Since $\mathcal{B}(G)$ is invariant under the braided group action, $B_{n+1}(S)$ is a subgroup of $B_{n+1}$. Let $\tilde{B}_{n+1}$ be the kernel of the composite

$$
B_{n+1} \longrightarrow \Sigma_{n+1} \stackrel{\text { sign }}{\longrightarrow} \mathbb{Z} / 2,
$$

that is, $\tilde{B}_{n+1}$ is the pre-image of the alternating group $A_{n+1}$.

Theorem 2.9. Let $G$ be a braided simplicial group. Then

1) $B_{n+1}\left(\mathcal{Z}(G)_{n}\right)=\tilde{B}_{n+1}$ or $B_{n+1}$;

2) $B_{n+1}\left(\mathcal{Z}(G)_{n}\right)=B_{n+1}$ if and only if $2 \cdot \pi_{n}(G)=0$.

Proof. Let $\sigma \in B_{n+1}\left(\mathcal{Z}(G)_{n}\right)$ and let $x \in \mathcal{Z}(G)_{n}$. By Lemma 2.8, we have

$$
\left(\sigma_{k}^{-1} \sigma \sigma_{k}\right)(x) \simeq\left(\sigma_{k} \sigma\right)\left(x^{-1}\right) \simeq \sigma_{k}\left(x^{-1}\right) \simeq x
$$

for each $k \geq-1$. Thus $B_{n+1}\left(\mathcal{Z}(G)_{n}\right)$ is a normal subgroup of $B_{n+1}$. By Lemma 2.8, we have

$$
\sigma_{s} \sigma_{t} \in B_{n+1}\left(\mathcal{Z}(G)_{n}\right)
$$

for any $s, t \geq-1$. It follows that $B_{n+1}\left(\mathcal{Z}(G)_{n}\right)=\tilde{B}_{n+1}$ or $B_{n+1}$, which is the assertion 1 .

If $B_{n+1}\left(\mathcal{Z}(G)_{n}\right)=B_{n+1}$, then by Lemma 2.8

$$
x \simeq x^{-1}
$$

for any $x \in \mathcal{Z}(G)_{n}$. Thus $2 \cdot \pi_{n}(G)=0$. Conversely, if $2 \cdot \pi_{n}(G)=0$, then

$$
\sigma_{k}(x) \simeq x
$$

for any $x \in \mathcal{Z}(G)_{n}$ and $k \geq-1$. Thus $B_{n+1}\left(\mathcal{Z}(G)_{n}\right)=B_{n+1}$. This shows assertion 2 .

Let $G$ be a braided simplicial group and let $H$ be a subgroup of $B_{n+1}$. Define

$$
G_{n}(H)=\left\{x \in G_{n} \mid \sigma(x) \simeq x \text { for all } \sigma \in H\right\}
$$

that is, $G_{n}(H)$ is the "homotopy" fixed set of $H$. Then $G_{n}(H)$ is a subgroup of $G_{n}$. By Theorem 2.9, we have that

$$
G_{n}\left(\tilde{B}_{n+1}\right) \supseteq \mathcal{Z}(G)_{n}
$$

Proposition 2.10. Let $G$ be a braided simplicial group and let $x \in G_{n}\left(\tilde{B}_{n+1}\right)$ with $n \geq 2$. Then

1) $d_{j}(x)=d_{j+2}(x)$ for each $j$;

2) $\sigma_{k} d_{j}(x)=d_{j+1}(x)$ for each $j, k$; 
3) $d_{j}(x)$ is a fixed point of $\tilde{B}_{n}$ for each $j$.

In particular, if $d_{j} x=1$ for some $j$, then $x \in \mathcal{Z}(G)_{n}$.

Proof. Since $x \in G_{n}\left(\tilde{B}_{n+1}\right)$, we have

$$
\sigma_{s} \sigma_{t}(x) \simeq x
$$

for any $s, t \geq-1$. It follows that

$$
\sigma_{-1} x \simeq \sigma_{0} x \simeq \sigma_{1} x \simeq \cdots \simeq \sigma_{n-2} x .
$$

Now for each $-1 \leq k \leq n-3$, we have

$$
d_{k+1} x=d_{k+2} \sigma_{k} x=d_{k+2} \sigma_{k+1} x=d_{k+3} x
$$

and so assertion 1 follows.

Now for each $0 \leq s \leq n-2$, we have

$$
d_{s+1}(x)=d_{s} \sigma_{s-1}(x)=d_{s} \sigma_{s}(x)=\sigma_{s-1} d_{s}(x) .
$$

Assertion 2 follows.

For any $s, t \geq-1$, we have

$$
\sigma_{s}\left(\sigma_{t} d_{0}(x)\right)=\sigma_{s}\left(d_{0}\left(\sigma_{t+1} x\right)\right)=d_{0}\left(\left(\sigma_{t+1} \sigma_{s+1}\right) x\right)=d_{0} x .
$$

Thus

$$
\sigma_{t} \sigma_{s}\left(d_{0}(x)\right)=d_{0}(x)
$$

for any $s, t \geq-1$ and so $d_{0}(x)$ is a fixed point of $\tilde{B}_{n}$. Since

$$
\sigma_{t} \sigma_{s}\left(d_{1}(x)\right)=\sigma_{s}\left(\left(\sigma_{-1} \sigma_{t}\right)\left(d_{0}(x)\right)\right)=\sigma_{s}\left(d_{0}(x)=d_{1}(x)\right.
$$

$d_{1}(x)$ is a fixed point of $\tilde{B}_{n+1}$ and hence assertion 3 .

Let $B_{n}$ be the subgroup of $B_{n+1}$ generated by $\sigma_{j}$ with $j \geq 0$. By inspecting the proof, we have

Proposition 2.11. Let $G$ be a braided simplicial group and let $x \in G_{n}\left(\tilde{B}_{n}\right)$ with $n \geq 2$. Then

1) $d_{j}(x)=d_{j+2}(x)$ for each $j \geq 1$;

2) $\sigma_{k} d_{j}(x)=d_{j+1}(x)$ for each $j, k \geq 1$;

3) $d_{j}(x)$ is a fixed point of $\tilde{B}_{n-1}$ for each $j$;

4) $d_{0}(x)$ is a fixed point of $\tilde{B}_{n}$.

In particular, if $d_{0} x=1$ and $d_{j} x=1$ for some $j \geq 1$, then $x \in \mathcal{Z}(G)_{n}$.

The following lemma is well-known. We give an elementary proof. Let $S$ be a subset of a group $G$. We write $\langle S\rangle$ for the subgroup of $G$ generated by $S$. 
Lemma 2.12. Let $w \in F\left(y_{0}, \cdots, y_{n}\right)$ with $n \geq 0$. Suppose that there is a positive integer $k$ such that $\sigma_{j}^{k}(w)=w$ for $0 \leq j \leq n-1$. Then $w$ lies in the subgroup generated by $y_{0} y_{1} \cdots y_{n}$. In addition, if $\sigma_{-1}^{k} w=w$ and $n \geq 1$, then $w=1$.

Proof. The proof is given by induction on $n$. The assertion is trivial for $n=0$. Let $n=1$. We may assume that $k=2 t$ is an even integer. Let $x_{0}=y_{1}^{-1}$ and let $x_{1}=y_{0} y_{1}$. Then $F\left(y_{0}, y_{1}\right)=F\left(x_{0}, x_{1}\right)$. Since $\sigma_{0}\left(y_{0}\right)=y_{1}$ and $\sigma_{0}\left(y_{1}\right)=y_{1}^{-1} y_{0} y_{1}$, we have $\sigma_{0}=\chi_{x_{1}}$ and so

$$
\sigma_{0}^{k}=\chi_{x_{1}}^{t}=\chi_{x_{1}^{t}}
$$

We can write $w$ as a reduced word in $F\left(y_{0}, y_{1}\right)=F\left(x_{0}\right) * F\left(x_{1}\right)$. Then

$$
w=x_{0}^{n_{1}} x_{1}^{l_{1}} \cdots x_{0}^{n_{s}} x_{1}^{l_{s}}
$$

where $n_{j} \neq 0$ for $2 \leq j \leq s$ and $l_{j} \neq 0$ for $1 \leq j \leq s-1$. Suppose that $w \notin\left\langle x_{1}\right\rangle$. There are two cases: $n_{1} \neq 0$ or $n_{1}=0$. If $n_{1} \neq 0$, then $x_{1}^{t} w \neq w x_{1}^{t}$. This contradicts to that $\chi_{x_{1}^{t}}(w)=w$. Otherwise, $n_{1}=0$ and $s>1$. Then $w=x_{1}^{l_{1}} x_{0}^{n_{2}} w^{\prime}$ and $x_{1}^{t+l_{1}} x_{0}^{n_{2}} w^{\prime} \neq$ $x_{1}^{l_{1}} x_{0}^{l_{2}} w^{\prime} x_{1}^{t}$. This contradicts to that $\chi_{x_{1}^{t}}(w)=w$. Hence $w \in\left\langle x_{1}\right\rangle=\left\langle y_{0} y_{1}\right\rangle$.

Now suppose that the assertion holds for $n-1$ with $n>1$. Since

$$
F\left(y_{0}, \cdots, y_{n}\right)=F\left(y_{0}, \cdots, y_{n-1}\right) * F\left(y_{n}\right)
$$

is a free product, we can write $w$ as a word

$$
w=y_{n}^{l_{0}} w_{1} y_{n}^{l_{1}} \cdots w_{t} y_{n}^{l_{t}}
$$

where $w_{j} \neq 1 \in F\left(y_{0}, \cdots, y_{n-1)}\right.$ and $l_{j} \neq 0$ for $1 \leq j \leq t-1$. Because $\sigma_{j}\left(y_{n}\right)=y_{n}$ for $j<n-1$, we have

$$
\sigma_{j}^{k}\left(w_{i}\right)=w_{i}
$$

for $1 \leq i \leq t$ and $0 \leq j \leq n-2$. Let $x=y_{0} y_{1} \cdots y_{n-1}$. By induction, we have

$$
w_{i} \in\langle x\rangle
$$

for $1 \leq i \leq t$ and so

$$
w \in\left\langle x, y_{n}\right\rangle \text {. }
$$

Let $q: F\left(y_{0}, y_{1}, \cdots, y_{n}\right) \rightarrow F\left(y_{n-1}, y_{n}\right)$ be the projection defined by $q\left(y_{j}\right)=1$ for $j<n-1$ and $q\left(y_{j}\right)=y_{j}$ for $j \geq n-1$. Then

$$
q \circ \sigma_{n-1}=\sigma_{n-1} \circ q .
$$

Since $\sigma_{n-1} w=w$, we have $\sigma_{n-1}(q(w))=q(w)$ and so

$$
q(w) \in\left\langle y_{n-1} \cdot y_{n}\right\rangle
$$

Because the restriction

$$
\left.q\right|_{\left\langle x, y_{n}\right\rangle}:\left\langle x, y_{n}\right\rangle \rightarrow F\left(y_{n-1}, y_{n}\right)
$$


is an isomorphism, we have

$$
w \in\left\langle x \cdot y_{n}\right\rangle=\left\langle y_{0} y_{1} \cdots y_{n}\right\rangle
$$

and hence the result.

Let $K_{n}$ be the pure braided group, that is, $K_{n}$ is that kernel of the canonical epimorphism $B_{n} \rightarrow \Sigma_{n}$. Let $B_{n+1}$ be the subgroup of $B_{n+2}$ generated by $\sigma_{j}$ for $j \geq 0$. Recall that

$$
\pi_{n+1}\left(F\left(S^{1}\right)\right)=\mathcal{Z}\left(F\left(S^{1}\right)\right)_{n+1} / \mathcal{B}\left(F\left(S^{1}\right)\right)_{n+1} .
$$

Consider the actions of two braided groups $B_{n+2}$ and $B_{n+1}$ on $F\left(S^{1}\right)_{n+1} / \mathcal{B}\left(F\left(S^{1}\right)\right)_{n+1}$. We have

Theorem 2.13. If $n \geq 2$, then in $F\left(S^{1}\right)_{n+1} / \mathcal{B}\left(F\left(S^{1}\right)\right)_{n+1}$,

1) the fixed set of the pure braided group $K_{n+1}$-action is

$$
\mathbb{Z} \oplus \pi_{n+1}\left(F\left(S^{1}\right)\right)
$$

2) the fixed set of $K_{n+2}$-action is

$$
\pi_{n+1}\left(F\left(S^{1}\right)\right)
$$

Proof. We show that the homotopy fixed set of $K_{n+1}$ on $F\left(S^{1}\right)_{n+1}$ is generated by $y_{-1}$ and $\mathcal{Z}\left(F\left(S^{1}\right)\right)_{n+1}$. Assertions 1 and 2 will follow from this statement. Let $w$ be a homotopy fixed point of $K_{n+1}$-action on $F\left(S^{1}\right)_{n+1}$. Since

$$
\sigma_{k}^{2} d_{0}=d_{0} \sigma_{k+1}^{2}
$$

for $k \geq-1$, we have

$$
\sigma_{k}^{2} d_{0}(w)=d_{0}(w)
$$

for each $k \geq-1$. By Lemma 2.12, we have

$$
d_{0}(w)=1
$$

Now for each $1 \leq j \leq n+1$, we have

$$
\sigma_{k}^{2} d_{j}=\left\{\begin{array}{ccc}
d_{j} \sigma_{k+1}^{2} & \text { if } & j \leq k+1 \\
d_{j} \circ \sigma_{j-1}^{-1} \circ \sigma_{j-2}^{2} \circ \sigma_{j-1} & \text { if } & j=k+2 \\
d_{j} \sigma_{k}^{2} & \text { if } & j>k+2 .
\end{array}\right.
$$

By Lemma 2.12, there exists integers $k_{1}, k_{2}, \cdots, k_{n+1}$ such that

$$
d_{j}(w)=y_{-1}^{k_{j}}
$$

for $1 \leq j \leq n+1$. Since $d_{k} y_{-1}=y_{-1}$ for $k>0$, we have

$$
y_{-1}^{k_{j}}=d_{j}\left(y_{-1}^{k_{j}}\right)=d_{j} d_{j} w=d_{j} d_{j+1} w=d_{j}\left(y_{-1}^{k_{j+1}}\right)=y_{-1}^{k_{j+1}}
$$

for $1 \leq j \leq n$ and so

$$
k_{1}=k_{2}=\cdots=k_{n+1} \text {. }
$$


Let $w^{\prime}=y_{-1}^{-k_{1}} w$. Then

$$
d_{j}\left(w^{\prime}\right)=1
$$

for each $0 \leq j \leq n+1$ and $\left.w^{\prime} \in \mathcal{Z}_{(} F\left(S^{1}\right)\right)_{n+1}$. This shows that $w$ lies in the subgroup generated by $y_{-1}$ and cycles $\mathcal{Z}\left(F\left(S^{1}\right)\right)_{n+1}$ and hence the result.

Note: In $F\left(S^{1}\right)_{n+1} / \mathcal{B}_{n+1}$, since any element in the homotopy group is a homotopy fixed point of $\tilde{B}_{n+2}$, the fixed set of $\tilde{B}_{n+1}$ is $\mathbb{Z} \oplus \pi_{n+1}\left(F\left(S^{1}\right)\right)$ and the fixed set of $\tilde{B}_{n+2}$ is $\pi_{n+1}\left(F\left(S^{1}\right)\right)$.

\section{Braided Representation of $F\left(S^{1}\right)$}

3.1. A Representation of $F\left(S^{1}\right)$. In this subsection, the ground ring $R$ is $\mathbb{Z}$ or $\mathbb{Z} / p$. Let $X$ be a pointed set. Let $A(X)$ be the algebra of non-commutative formal power series with variables in any finite subset of $X$ over $R$ modulo the single relation that $*=1$, where $*$ is the base point of $X$. Let $X$ be a pointed simplicial set. The simplicial algebra $A(X)$ is defined by applying the functor $A$ to $X$. Let $X=S^{1}$ be the simplicial circle. By using the methods in [11], we have

1) There is a choice of generators in $A\left(S^{1}\right)_{n+1}$ such that

$$
A\left(S^{1}\right)_{n+1}=A\left(x_{0}, x_{1}, \cdots, x_{n}\right)
$$

is the associated algebra of the non-commutative formal power series in variables $x_{0}, x_{1}, \cdots, x_{n}$ over $R$.

2) The simplicial structure on $A\left(S^{1}\right)$ is given by

$$
d_{j} x_{k}=\left\{\begin{array}{clc}
x_{k-1} & \text { for } & j \leq k \\
0 & \text { for } & j=k+1 \\
x_{k} & \text { for } & j>k+1
\end{array}\right.
$$

and

$$
s_{j} x_{k}=\left\{\begin{array}{cl}
x_{k+1} & \text { for } \quad j \leq k, \\
x_{k}+x_{k+1}+x_{k} x_{k+1} & \text { for } \quad j=k+1, \\
x_{k} & \text { for } \quad j>k+1
\end{array}\right.
$$

for $0 \leq j \leq n+1$, where

$$
x_{-1}=\left(1+x_{n-1}\right)^{-1}\left(1+x_{n-2}\right)^{-1} \cdots\left(1+x_{0}\right)^{-1}-1
$$

in $A\left(S^{1}\right)_{n}$.

Let the Braided group $B_{n+1}$ act on $A\left(S^{1}\right)_{n+1}$ as follows. 
For each $0 \leq k \leq n-1, \sigma_{k}: A\left(S^{1}\right)_{n+1} \rightarrow A\left(S^{1}\right)_{n+1}$ is an automorphism of algebras with

$$
\sigma_{k}\left(x_{j}\right)=\left\{\begin{array}{cccc}
x_{k+1} & \text { if } & j=k \\
\left(1+x_{k+1}\right)^{-1}\left(1+x_{k}\right)\left(1+x_{k+1}\right)-1 & \text { if } & j=k+1 \\
x_{j} & & \text { otherwise }
\end{array}\right.
$$

for $0 \leq k \leq n-1$.

Let $\sigma_{-1}$ be an automorphism of $A\left(S^{1}\right)_{n+1}$ defined by

$$
\sigma_{-1}\left(x_{0}\right)=\left(1+x_{0}\right)^{-1}\left(1+x_{-1}\right)\left(1+x_{0}\right)-1=\left(1+x_{0}\right)^{-1}\left(1+x_{n}\right)^{-1} \cdots\left(1+x_{1}\right)^{-1}-1
$$

and $\sigma\left(x_{j}\right)=x_{j}$ for $j>0$. The subgroup of the automorphism group of $A\left(S^{1}\right)_{n+1}$ generated by $\sigma_{j}$ for $-1 \leq j \leq n-1$ is the braid group $B_{n+2}$. Let

$$
e: F\left(S^{1}\right) \rightarrow A\left(S^{1}\right)
$$

be the canonical representation, that is

$$
e\left(y_{j}\right)=1+x_{j}
$$

for each $j$.

Proposition 3.1. The simplicial algebra $A\left(S^{1}\right)$ is a braided simplicial algebra, that is, the braided action satisfies the identities 1 and 0 in Proposition 2.1. Furthermore, the representation e: $F\left(S^{1}\right) \rightarrow A\left(S^{1}\right)$ is a braided representation, that is e commutes with the braid group action and the simplicial structure.

The proof is straight forward.

Since the representation $e: F\left(S^{1}\right) \rightarrow A\left(S^{1}\right)$ is faithful [6], we have

Proposition 3.2. Let $w \in F\left(S^{1}\right)_{n+1}$. Then

1) $w \in \mathcal{Z}\left(F\left(S^{1}\right)\right)_{n+1}$ if and only if $e(w)-1 \in \mathcal{Z}\left(A\left(S^{1}\right)\right)_{n+1}$;

2) $w \in N F\left(S^{1}\right)_{n+1}$ if and only if $e(w)-1 \in N A\left(S^{1}\right)_{n+1}$.

Let $w=x_{i_{1}}^{n_{1}} x_{i_{2}}^{n_{2}} \cdots x_{i_{t}}^{n_{t}}$ be a monomial in $A\left(x_{0}, \cdots, x_{n}\right)$. We call $w$ is non-degenerate if the set

$$
\left\{i_{1}, \cdots, i_{t}\right\}=\{0, \cdots, n\}
$$

that is each letter $x_{j}$ with $0 \leq j \leq n$ appears in $w$ at least once.

Theorem 3.3. Let $f$ be a series in $A\left(S^{1}\right)_{n+1}$. Then $f \in N A\left(S^{1}\right)_{n+1}$ if and only if $f$ is a formal series of non-degenerate monomials.

The assertion follows from the following lemma. 
Lemma 3.4. Let $f$ be a series in $A\left(S^{1}\right)_{n+1}$. Then

$$
f \in \bigcap_{j=1}^{i+1} \operatorname{Ker}\left(d_{j}\right)
$$

if and only if $f$ is a linear summation of monomials in which each $x_{j}$ appears at least once for $0 \leq j \leq i$.

Proof. The proof is given by induction on $i$. Let $i=0$. Since $d_{1}$ is the projection, $\operatorname{Ker}\left(d_{0}\right)$ is a two sided ideal generated by $x_{0}$. Suppose that the assertion holds for $i-1$ with $i>0$. Then there is a decomposition

$$
\bigcap_{j=1}^{i} \operatorname{Ker} d_{j}=C \oplus D,
$$

where $C$ is the set of series of monomials in which each $x_{j}$ appears at least once for $0 \leq j \leq i$. Since $d_{i+1}$ is the projection, we find that $\left.d_{i+1}\right|_{C}=0$ and $\left.d_{i+1}\right|_{D}$ is a monomorphism from $D$ to $A\left(S^{1}\right)_{n}$. This shows that

$$
\bigcap_{j=1}^{i+1} \operatorname{Ker} d_{j}=C
$$

and hence the result.

3.2. Formal Steenrod Operations and Higher Differentials. In this subsection, the ground ring $R$ is $\mathbb{Z}$ or $\mathbb{Z} / p$. Let $A\left(x_{0}, \cdots, x_{n}\right)_{i}$ be the sub $R$-module of $A\left(x_{0}, \cdots, x_{n}\right)$ generated by monomial of degree $i$. Let $f: A\left(x_{0}, \cdots, x_{n}\right) \rightarrow A\left(x_{0}, \cdots, x_{n-1}\right)$ be an $R$-linear map. We call $f$ is a homogenous map of degree $t$ if

$$
f\left(A\left(x_{0}, \cdots, x_{n}\right)_{i}\right) \subseteq A\left(x_{0}, \cdots, x_{n-1}\right)_{i+t}
$$

for each $q$. Let $V$ be the free $R$-module generated by $x_{0}, \cdots, x_{n}$.

Lemma 3.5. Let $f_{t}: V \rightarrow A\left(x_{0}, \cdots x_{n-1}\right)$ be a sequence of $R$-linear maps with $t \geq 0$ such that

$$
f_{t}(V) \subseteq A\left(x_{0}, \cdots, x_{n-1}\right)_{t}
$$

for each $t$. Then there exists a unique sequence of homogeneous maps $P_{f}^{t}: A\left(x_{0}, \cdots, x_{n}\right) \rightarrow$ $A\left(x_{0}, \cdots, x_{n-1}\right)$ such that

1) $\left.P_{f}^{t}\right|_{V}=f_{t}$ for each $t \geq 0$;

2) The anti-Cartan formula

$$
P_{f}^{t}(x y)=\sum_{i+j=t} P_{f}^{i}(y) P_{f}^{j}(x)
$$

hold for any $x, y \in A\left(x_{0}, \cdots, x_{n}\right)$. 
The proof is straight forward.

Let $\chi: A\left(x_{0}, \cdots, x_{n}\right)$ be the convolution, that is, $\chi$ is the anti-automorphism with $\chi\left(x_{i}\right)=-x_{i}$ for each $i$. Let

$$
\bar{d}_{0}=d_{0} \circ \chi=\chi \circ d_{0} .
$$

Then we have $\bar{d}_{0}\left(x_{j}\right)=x_{j-1}$ for $j>0$ and

$$
\bar{d}_{0}\left(x_{0}\right)=\left(1+x_{0}\right)\left(1+x_{1}\right) \cdots\left(1+x_{n-1}\right)-1 .
$$

Let

$$
\Delta_{s-1}=\sum_{0 \leq l_{1}<l_{2}<\cdots<l_{s} \leq n-1} x_{l_{1}} x_{l_{2}} \cdots x_{l_{s}} .
$$

for $1 \leq s \leq n$. For $0 \leq s \leq n-1$, let $\delta_{s}: V \rightarrow A\left(x_{0}, \cdots, x_{n-1}\right)$ be the $R$-linear map defined by

$$
\delta_{i}\left(x_{j}\right)=\left\{\begin{array}{ccc}
x_{j-1} & \text { for } & i=0, j>0 \\
0 & \text { for } & i>0, j>0 \\
\Delta_{i} & \text { for } & j=0
\end{array}\right.
$$

Let

$$
\delta=\left(\delta_{0}, \delta_{1}, \cdots, \delta_{n-1}, 0, \cdots, 0\right) .
$$

Proposition 3.6. The map $\bar{d}_{0}: A\left(x_{0}, \cdots, x_{n}\right) \rightarrow A\left(x_{0}, \cdots, x_{n-1}\right)$ is decomposed as

$$
\bar{d}_{0}=\sum_{i=0}^{\infty} P_{\delta}^{i} .
$$

Proof. By the anti-Cartan formula, the map

$$
\sum_{i=0}^{\infty} P_{\delta}^{i}
$$

is a well-defined anti-homomorphism of algebras. Since

$$
\bar{d}_{0}\left(x_{j}\right)=\sum_{i=0}^{\infty} P_{\delta}^{i}\left(x_{j}\right)
$$

for each $0 \leq j \leq n$, the assertion follows.

Let $q_{i}: A\left(x_{0}, \cdots, x_{n}\right) \rightarrow A\left(x_{0}, \cdots, x_{n}\right)$ be the composite

$$
A\left(x_{0}, \cdots, x_{n}\right) \stackrel{\text { proj. }}{\longrightarrow} A\left(x_{0}, \cdots, x_{n}\right)_{i} \longleftrightarrow A\left(x_{0}, \cdots, x_{n}\right)
$$

for $0 \leq i<\infty$. Let

$$
\Gamma_{t} F\left(S^{1}\right)_{n+1}=\left\{w \in F\left(S^{1}\right)_{n+1} \mid q_{i}(w)=0 \quad \text { for } \quad 0<i<t\right\}
$$


for $1 \leq t<\infty$. It is well-known [6] that $\left\{\Gamma_{t}\right\}$ is the descending central series if $R=\mathbb{Z}$ and descending $p$-central series if $R=\mathbb{Z} / p$. Let

$$
L\left(S^{1}\right)=\bigoplus_{t=1}^{\infty} \Gamma_{t}\left(F\left(S^{1}\right)\right) / \Gamma_{t+1}\left(F\left(S^{1}\right)\right) .
$$

If $R=\mathbb{Z}$, then $L\left(S^{1}\right)$ is the free simplicial Lie algebra over $\mathbb{Z}$ generated by $S^{1}$. If $R=\mathbb{Z} / p$, then $L\left(S^{1}\right)$ is the free restricted simplicial Lie algebra over $\mathbb{Z} / p$ generated by $S^{1}$. The simplicial structure $L\left(S^{1}\right)$ is as follows.

1) $L\left(S^{1}\right)_{n+1}=L\left(x_{0}, \cdots, x_{n}\right)$;

2) $d_{j}\left(x_{k}\right)=x_{k}$ for $k<j-1, d_{j}\left(x_{j-1}\right)=0$ and $d_{j}\left(x_{k}\right)=x_{k-1}$ for $k>j-1$, where $x_{-1}=-\left(x_{0}+x_{1}+\cdots+x_{n-1}\right) \in L^{u}\left(S^{1}\right)_{n}$ or $L\left(S^{1}\right)_{n}$.

3) $s_{j} x_{k}=x_{k}$ for $k<j-1, s_{j} x_{j-1}=x_{j-1}+x_{j}$ and $s_{j} x_{k}=x_{k+1}$ for $k>j-1$.

A Lie monomial $w=\left[\left[x_{i_{1}}, x_{i_{2}}, \cdots\right]^{p^{s}}\right.$ in $L^{u}\left(S^{1}\right)_{n+1}$ or $L\left(S^{1}\right)_{n+1}$ is called non-degenerate if the set

$$
\left\{i_{1}, \cdots, i_{t}\right\}=\{0,1, \cdots, n\} .
$$

By Theorem 3.3, we have

Corollary 3.7. The Moore chain complexes $N L\left(S^{1}\right)_{n+1}$ is the submodules of $L\left(S^{1}\right)_{n+1}$ spanned by non-degenerate Lie monomials, respectively.

The spectral sequence, which is denoted by $\left\{E^{r}\left(F\left(S^{1}\right)\right\}\right.$, induced by the descending $p$-central (integral descending central) series of $F\left(S^{1}\right)$ is called (integral) Adams spectral sequence of $F\left(S^{1}\right)$. By Curtis Theorem, this spectral sequence is convergent to $\pi_{*}\left(F\left(S^{1}\right) ; p\right)=\pi_{*+1}\left(S^{2} ; p\right)$ ( or $\pi_{*+1}\left(S^{2}\right)$ if we use integral descending central series). A description of higher differentials in the Adams spectral sequence is as follows.

Let $w \in F\left(S^{1}\right)_{n+1}$. Then

$$
e(w)=1+\sum_{i=1}^{\infty} q_{i}(w)
$$

in $A\left(x_{0}, \cdots, x_{n}\right)$. We simply write $(w)_{i}$ for $q_{i}(w)$. Let

$$
\alpha \in E_{t, *}^{1}\left(F\left(S^{1}\right)\right)=\pi_{*}\left(L_{t}\left(S^{1}\right)\right)
$$

and let $z^{\alpha}$ be a cycle in the simplicial group $L\left(S^{1}\right)$ such that the homotopy class of $z^{\alpha}$ is $\alpha$, that is $z^{\alpha}$ is a cycle representative of $\alpha$. Since the map

$$
g: \Gamma_{t}\left(F\left(S^{1}\right)\right) \rightarrow L_{t}\left(S^{1}\right)=\Gamma_{t}\left(F\left(S^{1}\right)\right) / \Gamma_{t+1}\left(F\left(S^{1}\right)\right)
$$

is a simplicial epimorphism, there is an element

$$
w^{\alpha} \in N\left(\Gamma_{t}\left(F\left(S^{1}\right)\right)\right)=N\left(F\left(S^{1}\right)\right) \cap F\left(S^{1}\right)
$$

such that

$$
g\left(w^{\alpha}\right)=z^{\alpha}
$$


The element $w^{\alpha}$ is called a Moore representative of $\alpha$.

Theorem 3.8. Let $\alpha \in E_{t, *}^{1}\left(F\left(S_{n+1}^{1}\right)\right)$ and let $w_{\alpha}$ be a Moore representative in $F\left(S^{1}\right)_{n+1}$. Let $1 \leq r \leq \infty$. Then $d^{j}(\alpha)=0$ for $j<r$ if and only if the following linear equations holds in $A\left(x_{0}, \cdots, x_{n-1}\right)$ :

$$
\sum_{i=0}^{j} P_{\delta}^{i} w_{t+j-i}^{\alpha}=0
$$

for $0 \leq j<r$. Furthermore, if $r<\infty$, then

$$
\sum_{i=0}^{r} P_{\delta}^{i} w_{t+r}^{\alpha} \in L_{t+r}\left(S^{1}\right)_{n} \subseteq A\left(x_{0}, \cdots, x_{n-1}\right),
$$

which is a cycle representative of $-d^{r}(\alpha)$.

Proof. Since $d^{r}(\alpha)=0$ if and only if

$$
d_{0}\left(w^{\alpha}\right) \in \Gamma_{t+r} F\left(S^{1}\right)_{n},
$$

the assertion follows from Proposition 3.6.

Let $w$ be a word in $F\left(S^{1}\right)_{n+1}$. We call $w$ is a basic non-degenerate commutator of weight $s=l(w)$ if $w$ can be written down as a commutator

$$
\left.\left[\cdots\left[y_{i_{1}}, y_{i_{2}}\right], \cdots\right], y_{i_{s}}\right]
$$

such that the set

$$
\left\{i_{1}, \cdots, i_{s}\right\}=\{0, \cdots, n\}
$$

that is each generator $y_{j}$ occurs in the commutator $w$ at least once. Let $N_{t} F\left(S^{1}\right)_{n+1}$ be the subgroup of $F\left(S^{1}\right)_{n+1}$ generated by basic nondegenerate commutators $w$ with $l(w) \geq t$ and let $N_{t}^{(p)} F\left(S^{1}\right)_{n+1}$ be the subgroup of $F\left(S^{1}\right)_{n+1}$ generated by $w^{p^{r}}$, where $w$ runs over all basic non-degenerated commutators with $l(w) \cdot p^{r} \geq t$. Note that

$$
N_{t} F\left(S^{1}\right)_{n+1} \subseteq N\left(F\left(S^{1}\right)\right)_{n+1} \cap \Gamma_{t}\left(F\left(S^{1}\right)_{n+1}\right)
$$

if $R=\mathbb{Z}$ and

$$
N_{t}^{(p)} F\left(S^{1}\right)_{n+1} \subseteq N\left(F\left(S^{1}\right)\right)_{n+1} \cap \Gamma_{t}\left(F\left(S^{1}\right)_{n+1}\right)
$$

if $R=\mathbb{Z} / p$.

By Corollary 3.7, we have

Proposition 3.9. Let $r$ be any non-negative integer. If $R=\mathbb{Z}$, there is an isomorphism

$$
\begin{gathered}
N_{t} F\left(S^{1}\right)_{n+1} /\left(N_{t} F\left(S^{1}\right)_{n+1} \cap \Gamma_{t+r} F\left(S^{1}\right)_{n+1}\right) \\
\cong N\left(F\left(S^{1}\right)\right)_{n+1} \cap \Gamma_{t}\left(F\left(S^{1}\right)\right)_{n+1} /\left(N\left(F\left(S^{1}\right)\right)_{n+1} \cap \Gamma_{t+r}\left(F\left(S^{1}\right)\right)_{n+1}\right) .
\end{gathered}
$$


If $R=\mathbb{Z} / p$, then there is an isomorphism

$$
\begin{gathered}
N_{t}^{(p)} F\left(S^{1}\right)_{n+1} /\left(N_{t}^{(p)} F\left(S^{1}\right)_{n+1} \cap \Gamma_{t+r} F\left(S^{1}\right)_{n+1}\right) \\
\cong N\left(F\left(S^{1}\right)\right)_{n+1} \cap \Gamma_{t}\left(F\left(S^{1}\right)\right)_{n+1} /\left(N\left(F\left(S^{1}\right)\right)_{n+1} \cap \Gamma_{t+r}\left(F\left(S^{1}\right)\right)_{n+1}\right) .
\end{gathered}
$$

Remark 3.10. By Theorem 3.8 and Proposition 3.9, $\pi_{*}\left(L_{t}\left(S^{1}\right)\right)$ are represented by those words $w$ in $N_{t} F\left(S^{1}\right)$ (or $N_{t}^{(p)} F\left(S^{1}\right)$ ) with $P_{\delta}^{0} w_{t}=0$ and the higher differentials in the Adams spectral sequence are related to higher formal Steenrod operations on $N_{t} F\left(S^{1}\right)$.

3.3. $E^{1}$-terms of The Integral Adams Spectral Sequence. In this subsection, the ground ring $R$ is a subring of $\mathbb{Q}$. Let $L$ be the free functor from free $R$-modules to Lie algebras. Let $X$ be a pointed simplicial set. Let $\bar{R}(X)=R(X) / R(*)$ be the reduced free simplicial $R$-module generated by $X$. In particular, $\bar{R}\left(S^{n}\right)=K(R, n)$. Let $L(X)=L(\bar{R}(X))$ for any pointed simplicial set $X$. Let $V$ be a free $R$-module and let $L^{\prime}$ be the kernel of the abelianizer

$$
L(V) \rightarrow V .
$$

Then $L^{\prime}$ is functor from free $R$-modules to graded free $R$-modules. Let $Q_{n}\left(L^{\prime}(V)\right)$ be the set of indecomposable elements of degree $n$ of $L^{\prime}(V)$. Let $S_{n}(V)$ be the set of monomials of degree $n$ in the polynomial algebra $S(V)$. Note that $Q_{1}\left(L^{\prime}(V)\right)=0$.

Lemma 3.11. For each $n \geq 2$, there is a functorial short exact sequence

$$
0 \rightarrow Q_{n}\left(L^{\prime}(V)\right) \rightarrow S_{n-1}(V) \otimes V \stackrel{\text { mult. }}{\longrightarrow} S_{n}(V) \rightarrow 0 .
$$

Proof. Let $K(V)$ be the kernel of $S_{n-1}(V) \otimes V \stackrel{\text { mult }}{\longrightarrow} S_{n}(V)$ and let $\phi: T_{n}(V) \rightarrow S_{n}(V)$ be the composite

$$
T_{n}(V)=T_{n-1}(V) \otimes V \stackrel{\text { proj. }}{\longrightarrow} S_{n-1}(V) \otimes V \stackrel{\text { mult. }}{\longrightarrow} S_{n}(V) .
$$

Then $\left.\phi\right|_{L_{n}^{\prime}(V)}: L_{n}^{\prime}(V) \rightarrow S_{n}(V)$ is zero which gives a functorial map

$$
\tilde{\phi}: L_{n}^{\prime}(V) \rightarrow K(V) \text {. }
$$

It is a routine work to show that the map $\tilde{\phi}$ is onto and $\left.\tilde{\phi}\right|_{D_{n}\left(L^{\prime}(V)\right)}: D_{n}\left(L^{\prime}(V)\right) \rightarrow$ $K(V)$ is zero and so $\tilde{\phi}$ factors through $Q_{n}\left(L^{\prime}(V)\right)$, where $D\left(L^{\prime}(V)\right)$ is the set of decomposable elements of $L^{\prime}(V)$. Thus $K(V)$ is a functorial quotient of $Q_{n}\left(L^{\prime}(V)\right)$. By checking Poincare series, the quotient map

$$
Q_{n}\left(L^{\prime}(V)\right) \rightarrow K(V)
$$

is an isomorphism and hence the result.

Note: There is no functorial cross-section from $Q\left(L^{\prime}\right)$ to $L^{\prime}$ (See $[8]$ ). 
Proposition 3.12. There is a homotopy equivalence

$$
L\left(S^{1}\right) \simeq \bar{R}\left(S^{1}\right) \oplus L\left(S^{2}\right) .
$$

Proof. Since $K(\mathbb{Z}, 1) \simeq S^{1}$, we have

$$
Q\left(L^{\prime}\right) \simeq \bar{R}\left(S^{2}\right)
$$

Let $\phi: L\left(L_{2}\left(S^{1}\right)\right) \rightarrow L^{\prime}\left(S^{1}\right)$ be the inclusion. Then $\phi$ is a homotopy equivalence by checking the spectral sequence induced by Lie filtrations. The assertion follows.

Let $V$ be a free simplicial $R$-module and let $C$ be a (pointed) simplicial coalgebra. Let $f, g: C \rightarrow T(V)$ be pointed simplicial coalgebra maps. We call $f$ is coalgebra homotopic to $g$ if there is a pointed homotopy $F_{t}: C \rightarrow T(V)$ such that $F_{0}=f$, $F_{1}=g$ and $F_{t}$ is a coalgebra map for each $t$. Let

$$
[T(V), T(V)]^{\text {coalg }}
$$

be the set of coalgebra homotopy classes. If $f: T(V) \rightarrow T(V)$ is a simplicial coalgebra map, then we have the restriction

$$
\left.f\right|_{L(V)}: L(V) \rightarrow L(V) .
$$

If $f$ is coalgebra homotopic to $g$, then

$$
\left.\left.f\right|_{L(V)} \simeq g\right|_{L(V)}
$$

This defines a map

$$
\theta:[T(V), T(V)]^{\text {coalg }} \rightarrow[L(V), L(V)] .
$$

Theorem 3.13. Suppose that $R=\mathbb{Z}_{(p)}$. Then the homotopy groups $\pi_{*}\left(L\left(S^{2 n}\right)\right)$ has the following exponents.

1) If $n=1,3$, then

$$
p \cdot \pi_{*}\left(L\left(S^{2 n}\right)\right)=0
$$

for $*>2 n$ and any prime $p$;

2) If $p=2$, then

$$
4 \cdot \pi_{*}\left(L_{t}\left(S^{2 n}\right)\right)=0
$$

for $t>2$ and any $n$.

Proof. Consider the Cohen representation

$$
\theta: H_{\infty} \rightarrow\left[T\left(S^{2 n}\right), T\left(S^{2 n}\right)\right]^{\text {coalg }} \rightarrow\left[L\left(S^{2 n}\right), L\left(S^{2 n}\right)\right],
$$

where $H_{\infty}$ is the Cohen group. First we assume that $n=1,3$. Then the Samelson product

$$
S^{2 n} \wedge S^{2 n} \rightarrow F\left(S^{2 n}\right)
$$


is null homotopic. Since $\pi_{*}\left(\Gamma_{2}\left(F\left(S^{2 n}\right)\right) \rightarrow \pi_{*}\left(F\left(S^{2 n}\right)\right)\right.$ is a monomorphism, the Samelson product

$$
S^{2 n} \wedge S^{2 n} \rightarrow \Gamma_{2}\left(F\left(S^{2 n}\right)\right)
$$

is null homotopic and so the composite

$$
S^{2 n} \wedge S^{2 n} \rightarrow \Gamma_{2}\left(F\left(S^{2 n}\right)\right) \rightarrow \Gamma_{2}\left(F\left(S^{2 n}\right) / \Gamma_{3}\left(F\left(S^{2 n}\right)\right)\right.
$$

is null homotopic. Let $J_{t} T\left(S^{2 n}\right)=\mathbb{Z}_{(p)}\left(J_{t}\left(S^{2 n}\right)\right)$. Then there is a monomorphism

$$
\left[J_{t} T\left(S^{2 n}\right), T\left(S^{2 n}\right)\right]^{\text {coalg }} \rightarrow\left[\mathbb{Z}_{(p)}\left(\left(S^{2 n}\right)^{\times t}\right), T\left(S^{2 n}\right)\right]^{\text {coalg }}
$$

for any $t$. Since the Samelson product is trivial, the group

$$
\left[\mathbb{Z}_{(p)}\left(\left(S^{2 n}\right)^{\times t}\right), T\left(S^{2 n}\right)\right]^{\text {coalg }}
$$

is abelian. Let $\mathrm{id}^{* p}: T(V) \rightarrow T(V)$ be the $p$-fold convolution product of the identity and let $T(p): T(V) \rightarrow T(V)$ be the morphism of Hopf algebras induced by the map $p: V \rightarrow V, \quad, x \rightarrow p x$. Then $\left.\operatorname{id}^{* p}\right|_{J_{t} T\left(S^{2 n}\right)}$ is coalgebra homotopic to $\left.T(p)\right|_{J_{t} T\left(S^{2 n}\right)}$ in

$$
\left[\mathbb{Z}_{(p)}\left(\left(S^{2 n}\right)^{\times t}\right), T\left(S^{2 n}\right)\right]^{\text {coalg }}
$$

for each $t$ and so

$$
\left.\operatorname{id}^{* p}\right|_{J_{t} T\left(S^{2 n}\right)} \simeq T(p)_{J_{t} T\left(S^{2 n}\right)}
$$

for each $t$. This $\mathrm{id}^{* p}$ is coalgebra homotopic to $T(p)$. Let $x \in L_{t}\left(S^{2 n}\right)$. Then

$$
\operatorname{id}^{* p}(x)=p x \quad T(p)(x)=p^{t} x .
$$

Thus

in $\left[L_{t}\left(S^{2 n}\right), L_{t}\left(S^{2 n}\right)\right]$ and so

$$
p^{t}-p=p\left(p^{t-1}-1\right)=0
$$

$$
p \cdot \pi_{*}\left(L_{t}\left(S^{2 n}\right)\right)=0
$$

for $t>1$. Since $L_{1}\left(S^{2 n}\right)=K\left(Z_{(p)}, 2 n\right)$, we proves assertion 1 .

The proof of assertion 2 is similar to assertion 1 , where one needs the fact that the Samelson product

$$
S^{2 n} \wedge S^{2 n} \rightarrow F\left(S^{2 n}\right)
$$

is of order 2 up to homotopy and the higher Samelson products are null homotopic localized at 2 .

Remark 3.14. $\pi_{*}\left(L\left(S^{n}\right) \otimes \mathbb{Z} / p\right)$ is known as a specific module over the $\Lambda$-algebra [1, 4, 10. By considering the Bockstein spectral sequence for $\pi_{*}\left(L\left(S^{n}\right)\right)$, this theorem shows that $\pi_{*}\left(L\left(S^{2 n}\right)\right.$ is the kernel of the Bockstein on $\pi_{*}\left(L\left(S^{2 n}\right) \otimes \mathbb{Z} / p\right)$ when $n=1,3$.

Theorem 3.15. Let $R=\mathbb{Z}_{(p)}$. If $t$ is not a power of $p$, then

$$
\pi_{*}\left(L_{t}\left(S^{2 n}\right)\right)=0 .
$$


Proof. By a result in [8], there exist functors $A^{\min }$ and $Q_{t}^{\max }$ for $t \geq 2$ such that

1) $A^{\text {min }}$ is a (smallest) coalgebra retract of $T$ with $V \subseteq A^{\min }(V)$ for each $V$;

2) $Q_{t}^{\max }$ is a subfunctor of $L_{t}$ and $Q_{t}^{\max }$ is a retract of $T_{t}$;

3) There is a functorial coalgebra decomposition

$$
T(V) \cong T\left(\bigoplus_{t=2}^{\infty} Q_{t}^{\max }(V)\right) \otimes A^{\min }(V) .
$$

Let $L^{\min }(V)$ be the primitives of $A^{\min }(V)$. Then there is a functorial decomposition

$$
L(V) \cong L\left(\bigoplus_{t=2}^{\max } Q^{\max }(V)\right) \oplus L^{\min }(V) .
$$

Since $Q_{t}^{\max }$ is a retract of $T_{t}, Q^{\max }\left(S^{2 n}\right)$ is a retract of $T_{t}\left(S^{2 n}\right)$ and so $Q_{t}^{\max }\left(S^{2 n}\right)$ is either contractible or homotopic to $K\left(\mathbb{Z}_{(p)}, 2 t n\right)$. Now $Q_{t}^{\max }\left(S^{2 n}\right)$ is contractible because $Q_{t}^{\max }$ is subfunctor of $L_{t}(V)$. It follows that

$$
L\left(\bigoplus_{t=2}^{\infty} Q_{t}^{\max }\left(S^{2 n}\right)\right)
$$

is contractible. The assertion follows from a result in [8] that $L_{t}$ is a functorial retract of

$$
L\left(\bigoplus_{t=2}^{\infty} Q_{t}^{\max }\left(S^{2 n}\right)\right)
$$

if $t$ is not a power of $p$.

By Theorems 3.13 and 3.15, we have

Theorem 3.16. Let $\left\{E^{r}\right\}_{r \geq 1}$ be the integral Adams spectral sequence of $F\left(S^{1}\right)$. Then

1) $E_{t, *}^{r}=0$ unless $t=2 p^{s}$ some prime $p$ and some non-negative integer $s$.

2) $p \cdot E_{2 p^{s}, *}^{r}=0$ for any prime $p$ and any integer $s>0$.

3) Let $\alpha \in E_{2 p^{s}, *}^{1}$ with $s>0$. Then the only differentials $d^{r}$, which are possibly non-trivial on $\alpha$, are

$$
d^{2 p^{t}-2 p^{s}}
$$

with $t>s$.

\section{REFERENCES}

[1] Bousfield, Curtis, Kan, Quillen, Rector and Schlesinger, The mod-p lower central series and the Adams spectral sequence, Topology 5 (1966), 331-342.

[2] F. R. Cohen, Cohomology of braid groups, Bull. Amer. Math. Soc. 79 (1973), 761-764.

[3] F. Cohen, On combinatorial group theory in homotopy, Contemp. Math., 188 (1995), 57-63. 
[4] E. B. Curtis, Simplicial homotopy theory, Advancs in Math. (1971), 107-209.

[5] E. B. Curtis and M. Mahowald, The unstable Adams spectral sequence for $S^{3}$, Contemp. Math., 96 (1989), 125-162.

[6] W. Magnus, A. Karrass and D. Solitar, Combinatorial group theory, Interscience Publishers (1966).

[7] J. C. Moore, Homotopie des complexes monöideaux, Seminaire Henri Cartan (1954-55).

[8] Paul Selick and Jie Wu, On natural decompositions of loop suspensions and natural coalgebra decompositions of tensor algebras, Memoirs AMS, to appear.

[9] H. Toda, Composition methods in homotopy groups of spheres, Princeton Univ. Press, 1962.

[10] Robert Wellington, The unstable Adams spectral sequence for free iterated loop spaces, Memoirs AMS, 36, no. 258, (1982).

[11] Jie Wu, Combinatorial descriptions of the homotopy groups of certain spaces, Math. Proc. Camb. Philos. Soc. to appear.

Department of Mathematics, National University of Singapore, Singapore 117543 , Republic of Singapore, Matwuj@Nus.Edu.sG 